\title{
EUS in localizing safe alternate access sites for natural orifice transluminal endoscopic surgery: initial experience in a porcine model
}

\author{
B.J. Elmunzer · S.J. Schonmisch · J.A. Trunzo - B.K. Poulose · C.P. Delaney - M.F. McGee et al. \\ Gastrointest Endosc 2009;69:108-14 \\ Commentaire d'article du Pr F. Prat
}

(C) Springer-Verlag 2009

La chirurgie endoscopique transluminale per-orificielle, mieux connue par son acronyme anglais « NOTES » (Natural Orifice Transluminal Endoscopic Surgery), fait couler de l'encre et excite les imaginations, même si son intérêt et sa place future ne sont pas encore bien définis. Dans une conception réellement endoscopique de cette méthode, où l'abord de la cavité abdominale se ferait à l'aide d'un endoscope souple par perforation de la paroi gastrique ou rectosigmoïdienne, le choix d'un point d'entrée adéquat et sans danger est fondamental. Actuellement, les techniques d'abord aveugle par perçage de la paroi gastrique par exemple, ne sont envisageables qu'après création d'un pneumopéritoine, qui est la première étape d'une laparoscopie. En l'absence de cette technique, l'échoendoscopie peut être précieuse pour choisir une fenêtre d'entrée.

Un travail conjoint des universités de Cleveland et Ann Harbor aux USA (1) s'est intéressé aux différentes voies d'abord trans-gastriques et trans-rectales, dans le modèle porcin, qui se prête parfaitement à la simulation des interventions NOTES abdominales. Les accès transluminaux ont été obtenus soit par ponction diathermique, soit par ponction à l'aiguille de 19G, grâce à un échoendoscope linéaire classique (champ ultrasonore latéral) ou à un prototype d'échoendoscope interventionnel à champ axial. Trente-deux procédures d'accès à travers l'estomac ou le rectum ont été réalisées chez 12 porcs, et réparties a priori selon leur caractère « sûr » ou non. Le caractère sûr (safe) était défini comme une ponction réalisée sous contrôle échoendoscopique, sans interposition d'un organe plein ou d'un vaisseau; les ponctions «non sûres » (unsafe) avaient pour but d'estimer les risques de lésions induites par des ponctions faites à l'aveugle; une

F. Prat $(\bowtie)$

Hôpital Cochin, F-75679 Paris cedex 14, France

E-mail : frederic.prat@cch.aphp.fr fois obtenu le passage d'un guide dans le péritoine ou le rétro-péritoine, la méthode d'introduction de l'endoscope était identique. Toutes les ponctions à l'aveugle (« unsafe ») entraînaient des complications, parfois graves comme des lacérations du foie ou une plaie de l'artère iliaque. Au contraire, 13 des 16 ponctions dites « safe »n'amenaient aucun incident avec 3 complications sur 16 ponctions dites «safe ». Deux des 3 complications observées étaient mineures et la troisième était une perforation d'une anse grêle.

La méthodologie de cette étude est discutable puisqu'elle définit a priori des conditions expérimentales qui vont permettre de montrer l'intérêt de l'échoendoscopie, sans effectuer de comparaison dans des conditions identiques. De plus, les points de ponction ne sont pas décrits avec précision et semblent différer entre les 2 groupes. Cependant, son objectif est important car la définition très stricte des conditions techniques d'un geste NOTES est nécessaire à l'éventuelle diffusion de ces techniques. En particulier, la prise rigoureuse de repères anatomiques endoscopiques et/ou échoendoscopiques est certainement un point crucial qui doit être défini pour chaque type d'intervention (abord intra ou rétropéritonéal, pelvien ou sus-mésocolique, etc.). L'intérêt de l'échoendoscopie est probable, en particulier pour une voie rétro-péritonéale. Il devra faire l'objet d'études plus systématiques mais il implique néanmoins la nécessité d'employer (au moins) deux endoscopes pour mener à bien une intervention NOTES par le gastroentérologue interventionnel.

\section{Référence}

1. Elmunzer BJ, Schomisch SJ, Trunzo JA, Poulose BK, Delaney CP, McGee MF, et al. EUS in localizing safe alternate access sites for natural orifice transluminal endoscopic surgery: initial experience in a porcine modelGastrointest Endosc 2009;69:108-14. 